\title{
SENSIBILIDADE AO RACHAMENTO DE BAGAS DAS VIDEIRAS 'CONCORD', 'ISABEL’ E 'BRS RÚBEA'
}

\author{
ROGÉRIO DE SÁ BORGES², SERGIO RUFFO ROBERTO³, FABIO YAMASHITA4, \\ JULIANA BONAMETTI OLIVATO ${ }^{5}$, ADRIANE MARINHO DE ASSIS ${ }^{6}$
}

RESUMO-Com o objetivo de avaliar a sensibilidade ao rachamento de frutos, foram realizados diferentes testes de laboratório com bagas de uva para processamento das cultivares Concord, Isabel e BRS Rúbea. Para a determinação do índice de rachamento, bagas maduras foram imersas em água destilada e avaliadas a cada hora, durante um período de dez horas. Com o auxílio de um texturômetro, foram realizados testes mecânicos. Em testes de compressão, foram avaliadas, no momento de rompimento das bagas, pressão de compressão, energia de deformação e força máxima de compressão. Com uma sonda de menor calibre, as bagas foram perfuradas, obtendo-se a força máxima de perfuração e deformação de perfuração no rompimento da película. Em testes de relaxação, foram avaliados o grau de solidez e a velocidade inicial de decaimento. Foram avaliadas também as correlações entre o índice de rachamento, e os testes mecânicos. Tanto o índice de rachamento quanto os testes mecânicos apontaram para a cultivar BRS Rúbea como a mais resistente ao rachamento e a cultivar Concord como a mais suscetível. A 'Isabel' apresentou resistência intermediária. O índice de rachamento, apresentou forte correlação com as variáveis avaliadas nos testes mecânicos e possui bom potencial para a avaliação da sensibilidade ao rachamento de materiais distintos de videira.

Termos para indexação: uva, índice de rachamento, compressão, perfuração, relaxação.

\section{SENSIVITY TO BERRY CRACKING OF 'CONCORD', 'ISABEL’ AND 'BRS RÚBEA' GRAPEVINES}

\begin{abstract}
In order to evaluate the sensitivity of fruit cracking, different laboratory tests were performed in grape berries of the processing varieties Concord, Isabel and BRS Rubea. To determine the cracking index, ripe berries were dipped in distilled water and evaluated every hour during a period of ten hours. Mechanical tests were performed using a texturometer. In compression tests, at the cracking time, pressing pressure, strain energy, maximum force of compression and compression strain were evaluated. With a smaller diameter probe, berries were drilled to the breakup of the skin resulting in maximum force of drilling and drilling strain. In stress relaxation tests the degree of solidity and initial stress decay rate were evaluated. The correlations between the cracking index and mechanical testing were evaluated too. Both the cracking index and mechanical testing pointed to the BRS Rúbea as the most resistant to cracking and the Concord cultivar as the most susceptible. Isabel presented intermediate resistance. The cracking index showed strong correlation with the parameters evaluated in the mechanical tests and has good potential for assessing the sensitivity to cracking of different grape cultivars.
\end{abstract}

Index terms: grape, cracking index, compression, drilling, relaxation.

\footnotetext{
1'(Trabalho 064-12). Recebido em: 10-01-2012. Aceito para publicação em: 27-06-2012.

${ }^{2}$ Engenheiro Agrônomo, Doutorando em Agronomia, Programa de Pós-Graduação do Centro de Ciências Agrárias (CCA), Universidade Estadual de Londrina (UEL), Rod. Celso Garcia Cid PR 445, Km 380. CEP 86051-990, CP 6001, Londrina-PR. E-mail: rborges@cnpso.embrapa.br

${ }^{3}$ Engenheiro Agrônomo, Dr. Professor Associado, Departamento de Agronomia/Fitotecnia, Universidade Estadual de Londrina (UEL), Bolsista do CNPq. Rod. Celso Garcia Cid PR 445, Km 380. CEP 86051-990, CP 6001, Londrina-PR. E-mail: sroberto@uel.br

${ }^{4}$ Engenheiro de Alimentos, Dr. Professor Associado, Departamento de Ciência e Tecnologia de Alimentos, Universidade Estadual de Londrina (UEL), Bolsista do CNPq. Rod. Celso Garcia Cid PR 445, Km 380. CEP 86051-990, CP 6001, Londrina-PR. E-mail: fabioy@uel.br

${ }^{5}$ Farmacêutica Industrial, Doutorando em Ciência de Alimentos, Programa de Pós-graduação do Centro de Ciências Agrárias (CCA), Universidade Estadual de Londrina (UEL), Rod. Celso Garcia Cid PR 445, Km 380. CEP 86051-990, CP 6001, Londrina-PR. E-mail: jubonametti@gmail.com

${ }^{6}$ Engenheira Agrônoma, Dra. Professora Assistente, Departamento de Agronomia/Fitotecnia, Universidade Estadual de Londrina (UEL), Bolsista da Capes. Rod. Celso Garcia Cid PR 445, Km 380. CEP 86051-990, CP 6001, Londrina- PR. E-mail: agroadri@ig.com.br
} 


\section{INTRODUÇÃO}

O rachamento é um problema de muitas espécies de frutos de casca fina e macia como uva, cereja, maçã, pêssego, pera e tomate. Existem diferenças varietais quanto à suscetibilidade ao rachamento, e os critérios de classificação muitas vezes são de natureza subjetiva, definindo grupos de baixa, média ou alta suscetibilidade.

Na cultura da videira, o rachamento das bagas na maturação provoca perdas tanto em uvas para mesa como para processamento, reduzindo a qualidade e a produção. Esse problema vem sendo estudado por diferentes perspectivas em que o componente genético se destaca, uma vez que comumente são verificadas diferenças entre as cultivares de uva quanto à suscetibilidade ao rachamento (LANG; DÜRING, 1990). Fatores ambientais, como a ocorrência de chuvas na época de colheita e a disponibilidade de água no solo estão relacionados com o rachamento das bagas (LULU et al., 2005). Segundo Mello (2009), chuvas acima da média durante a colheita provocaram perdas de até $40 \%$ em uvas para mesa na região do Vale do São Francisco,enquanto Clarke et al. (2010) observaram que chuvas excessivas causaram perdas por rachamento em 'Cabernet Sauvignon', 'Shiraz' e 'Riesling'.

As principais cultivares para processamento produzidas no Brasil são ‘Isabel’, 'Concord’ e ‘Bordô', sendo a cultivar Isabel responsável pela maior área de produção (SATO et al., 2008). A cultivar Concord origina produto de melhor qualidade, podendo ser utilizada tanto sozinha como em cortes. Quanto ao rachamento, 'Isabel' e 'Bordô' são consideradas medianamente resistentes, e a cultivar Concord, muito suscetível. Mais recentemente, novos materiais foram lançados com melhores características, principalmente quanto à coloração, teor de sólidos solúveis e resistência ao rachamento de bagas, dentre eles a 'BRS Rúbea', 'BRS Cora', 'BRS Violeta' e 'BRS Carmem’ (CAMARGO et al., 2010).

Do ponto de vista fisiológico, o rachamento na maturação ocorre pelas variações no volume das bagas ocasionado pelo fluxo de água para dentro e para fora das bagas. Estudos demonstraram que as bagas possuem elasticidade que lhes permite, até certo limite, suportar essas variações, e essa capacidade pode variar entre cultivares (CONSIDINE; KRIEDEMANN, 1972; CONSIDINE; BROWN, 1981; BERNSTEIN; LUSTIG, 1985).

O método mais evidente para se verificar a suscetibilidade varietal ao rachamento seria em condições de campo. No entanto, uma vez que o rachamento em campo depende diretamente do estádio de maturação e das condições climáticas, é difícil obter condições idênticas para diferentes cultivares, e os dados de rachamento obtidos em uma única safra refletem muito mais uma distribuição acidental do que uma real sensibilidade varietal.

$\mathrm{Na}$ tentativa de verificar diferenças entre distintas cultivares ao rachamento e estudar suas causas, alguns autores estudaram métodos que pudessem representar o que se observa no campo para diferentes cultivares. Yamamoto e Satoh (1994) adaptaram para videira um método descrito por Verner e Blodgett (1936) para cerejas, determinando o índice de rachamento por imersão das bagas de uva em água destilada. Outros autores avaliaram a relação do rachamento com aspectos histológicos (HANKINSON et al., 1977), estruturais (CONSIDINE, 1981 e 1982; MATTHEWS et al., 1987) e mecânicos (CONSIDINE; KRIEDEMANN, 1972; BERNSTEIN; LUSTIG, 1985; LETAIEF et al., 2006).

Existem poucos trabalhos de determinação da sensibilidade ao rachamento das principais cultivares de uva para processamento produzidas no Brasil (YAMAMOTO; SATOH, 1994; FAVA et al., 2011). Estudos dessa natureza, com a utilização de parâmetros objetivos de avaliação do rachamento, poderão auxiliar na seleção de cultivares mais resistentes e contribuir para a definição das áreas de cultivo e do manejo mais adequado para cada material, reduzindo os riscos de perdas. Dessa forma, o objetivo deste trabalho foi avaliar a suscetibilidade ao rachamento de bagas das uvas 'Concord', 'Isabel' e 'BRS Rúbea’, utilizando-se de testes de imersão e de testes mecânicos de compressão, perfuração e relaxação, verificando-se possíveis correlações entre eles.

\section{MATERIAL E MÉTODOS}

Foram avaliadas na safra de 2010/2011 bagas de uva das cultivares Concord (Vitis labrusca), Isabel (V. labrusca) e BRS Rúbea ((V. labrusca x (V. labrusca x $V$. vinifera)) x $V$. labrusca) enxertados sobre porta-enxerto 'IAC 766-Campinas' (Riparia do Traviú x Vitis caribaea). As plantas foram conduzidas em sistema de latada, no espaçamento de 4,0 m x 2,0 $\mathrm{m}$, e encontravam-se no sexto ano de produção, em área experimental do Centro Tecnológico da Corol Cooperativa Agroindustrial, localizado no município de Rolândia-PR, (23 27’ S, 51²7’ O), com 600 m de altitude, precipitação média anual de 1.610 mm e temperatura média anual de $21,2^{\circ} \mathrm{C}$. As precipitações acumuladas nos meses de dezembro de 2010 e janeiro de 2011, período que compreende do início ao final da maturação, foram de 116,8 e 154,7 mm,respectivamente. Com o objetivo de padronizar a coleta das 
amostras, a evolução da maturação foi acompanhada por meio de análises químicas realizadas semanalmente para cada cultivar, do início do amolecimento das bagas até a colheita. O teor de sólidos solúveis (SS) foi determinado utilizando-se um refratômetro digital de bancada com compensação de temperatura (Modelo DR 301-95, Krüss Optronic, Alemanha), através do esmagamento e posterior filtragem das amostras em algodão, e o resultado, expresso em ${ }^{\circ}$ Brix. As determinações da acidez titulável (AT) do mosto foram realizadas em titulador potenciométrico digital (Modelo Tritoline Easy, Schott Geräte, Alemanha), com pH final ajustado para 8,2. A acidez foi obtida por titulação do mosto com solução padronizada de $\mathrm{NaOH}$, e o resultado expresso, em porcentagem de ácido tartárico (Tabela 1). Para os testes de sensibilidade ao rachamento, a coleta das amostras deu-se com valores de SS ao redor de $13,0^{\circ}$ Brix.

Para a realização de cada teste, foram colhidos cachos maduros na primeira hora da manhã (entre 6h e 7h30) para garantir a máxima turgescência das bagas, e, após a colheita, os cachos foram acondicionados em sacos de polietileno transparente e transportados sob refrigeração.

Como forma de evitar a perda de turgescência das bagas de uva desde o campo até o laboratório, foi introduzido no pedúnculo dos cachos recém-cortados um segmento de espuma fenólica saturada com água. A metodologia foi definida com base em um pré-teste, em que se utilizou a técnica da aplanação, desenvolvida por Bernstein e Lustig (1981 e 1985) e adaptada por Calbo e Nery. (1995), para avaliar a turgescência das bagas. A técnica consiste em colocar cada baga em um suporte vertical e apoiar uma cuba de vidro de massa conhecida sobre a baga. A turgescência é medida como a razão entre a massa da cuba e a área da baga amassada sob a cuba. No pré-teste, foi utilizado como cuba de vidro um Becker de $180 \mathrm{~g}$. Foram colhidos cachos com e sem a introdução do segmento de espuma fenólica, e a turgescência das bagas, avaliada pela área aplanada no campo, no momento da colheita e quatro horas após a colheita, no laboratório. A partir dos valores de área aplanada, calcularam-se os valores de pressão de turgescência, sendo as médias comparadas pelo teste de Tukey, a 5\%.

Para a determinação do índice de rachamento, utilizou-se o método desenvolvido por Verner e Blodgett (1936) para cerejas e adaptado por Yamamoto e Satoh (1994) para videiras. Para esse teste, o delineamento experimental foi em blocos casualizados, sendo dois blocos e 50 repetições. Cada bloco foi constituído de uma planta da qual foram colhidos cinco cachos ao acaso para a retirada das 50 bagas com pedicelo que constituíram as repetições. As bagas foram imersas em recipientes (750 ml) com água destilada contendo o surfactante Agral $^{\circledR}$ a $0,1 \%(\mathrm{v} / \mathrm{v})$ do produto comercial. A cada hora, e por um período de dez horas, as bagas com rachaduras visíveis foram retiradas do recipiente e contadas. O número de bagas rachadas em cada hora foi multiplicado por um fator de ponderação, neste caso, pelo número de horas restantes para completar dez horas de teste, pois desta forma as bagas que racham nas primeiras horas contribuem mais para o índice do que as que racham após um período maior de imersão. Para o cálculo do índice de rachamento, os valores ponderados foram somados e divididos por 500, que corresponde ao valor máximo possível de bagas rachadas, ou seja 50 bagas rachadas na primeira hora vezes 10 horas restantes do teste. O resultado foi expresso em porcentagem (VERNER; BLODGETT, 1936).

Para a avaliação das propriedades mecânicas dos frutos, foram realizados testes de compressão, perfuração e relaxação com auxílio de um texturômetro (Stable Micro System, modelo TATX2i, Inglaterra). Para esses testes, o delineamento experimental foi em blocos casualizados, sendo dois blocos e dez repetições. Cada bloco foi constituído de uma planta, da qual foram colhidos cinco cachos ao acaso para a retirada das 10 bagas com pedicelo.

No teste de compressão, cada baga foi colocada sobre a base do texturômetro e comprimida com uma sonda cilíndrica com ponta de seção plana de 35 bmm de diâmetro e paralela à base. A compressão deu-se no sentido da porção equatorial das bagas até $8 \mathrm{~mm}$, com força constante de $0,05 \mathrm{~N}$, à velocidade de $1 \mathrm{~mm} / \mathrm{s}$, suficiente para provocar o rompimento da película. Foram obtidas dessa forma as medidas de força máxima $(\mathrm{N})$ no rompimento e a deformação (\%). A partir dessas leituras, foram calculadas a pressão de compressão $(\mathrm{kPa})$, pela razão entre a força máxima, e a área da baga na porção equatorial $\left(\mathrm{mm}^{2}\right)$, e a energia de deformação (N.mm) pela área abaixo da curva força-deformação.

Para o teste de perfuração, foi usado o mesmo procedimento, porém com uma sonda com ponta tipo agulha de 2 mm de diâmetro. Foram obtidas as medidas de força máxima no momento da perfuração da baga pela sonda e de deformação (\%).

No teste de relaxação, as bagas foram comprimidas a $2 \mathrm{~mm}$, sem que houvesse rompimento, e mantidas sob compressão por 30 s. Dessa forma, foram obtidas medidas de força durante a compressão até a retirada da sonda.

Os dados de relaxação foram normalizados e analisados de acordo com o modelo empírico proposto por Peleg (1979), através da normalização da 
força $(\mathrm{F})$ através de um parâmetro de declínio $\mathrm{F}(\mathrm{t})$ (Equação 1) :

$$
\frac{F(t)}{F_{0}}=1-\frac{C_{1} \cdot t}{C_{2}+t}
$$

em que $F(t)$ é a força no tempo $t ; F_{0}$ é a força no tempo zero, e t é o tempo em segundos. $\mathrm{C}_{1}$ e $\mathrm{C}_{2}$ são os parâmetros do modelo que foram estimados por regressão não linear, utilizando o software STATISTICA 7.0 (STATSOFT, 2002). No modelo, 1-C $\mathrm{C}_{1}$ e $\mathrm{C}_{1} / \mathrm{C}_{2}$ fornecem informações sobre as características viscoelásticas do material. $\mathrm{O}$ valor de $1-\mathrm{C}_{1}$ é o grau de solidez, e a razão $C_{1} / C_{2}$ representa a taxa inicial de decaimento da força.

Para a realização de cada um dos testes descritos, foram determinados os diâmetros, individuais de cada baga amostrada. Devido à baixa variabilidade observada nos diâmetros foi utilizado para os cálculos dos resultados das variáveis analisadas o valor médio de $18 \mathrm{~mm}$ para todos os materiais avaliados.

\section{RESULTADOS E DISCUSSÃO}

A eficiência do uso da espuma fenólica para evitar a perda de turgescência das bagas foi comprovada pelos resultados obtidos no teste de aplanação. Os valores médios de pressão de turgescência medidos no campo $\left(0,37 \mathrm{kgf} . \mathrm{cm}^{2-1}\right)$ foram semelhantes aos obtidos nos cachos mantidos com a espuma fenólica $\left(0,36 \mathrm{kgf} . \mathrm{cm}^{2}{ }^{-1}\right)$. As médias de pressão das bagas oriundas dos cachos sem a espuma apresentaram valores significativamente menores $\left(0,24 \mathrm{kgf} . \mathrm{cm}^{2}{ }^{-1}\right)$, comprovando a eficiência do método.

Os índices de rachamento obtidos no teste de imersão são apresentados na Tabela 2. A cultivar Concord apresentou o maior índice (13,5\%), seguida pela 'Isabel' (6,3 \%), não sendo verificado rachamento das bagas da cultivar BRS Rúbea. Os resultados estão de acordo com observações de campo em que, mesmo em anos chuvosos, a cultivar BRS Rúbea tem apresentado alta resistência, superando até mesmo cultivares com moderada resistência, como á 'Isabel' (U. A. CAMARGO, comunicação pessoal). Com relação à cultivar Concord, os resultados também estão de acordo com observações de campo para esse material (NACHTIGAL; SCHNEIDER, 2007). A correlação do índice de rachamento com observações de campo também foi descrita por Considine (1982), que verificou semelhanças na intensidade e no padrão de rachamento. Yamamoto e Satoh (1994), em teste de imersão de bagas, obtiveram índice de rachamento de 5\% para a cultivar Concord.

No teste de imersão, as bagas maduras de uva sorvem água, aumentando seu volume e sua pressão de turgescência. Dependendo da quantidade de água sorvida e das características da cultivar, a película pode romper-se causando rachamento semelhante ao observado no campo, quando ocorrem chuvas no final da maturação. Alguns trabalhos comprovam essa capacidade de as bagas de uva sorverem água e aumentarem seu volume e pressão de turgescência, quando imersas em água (CONSIDINE; KRIEDEMANN, 1972; CONSIDINE, 1982; BERNSTEIN; LUSTIG, 1985; YAMAMOTO; SATOH, 1994).

A sorção de água pelas bagas de uva está diretamente ligada ao seu potencial osmótico, pois elas funcionam como osmômetros devido às características semipermeáveis de sua película (CONSIDINE; KRIEDEMANN, 1972). Esse potencial osmótico, por sua vez, depende essencialmente do teor de sólidos solúveis, cujos valores possuem forte correlação ( $\mathrm{r}$ $=0,99$ ) (MATTHEWS et al., 1987; THOMAS et al., 2008). Uma vez que os teores de sólidos solúveis totais não diferiram entre si para as cultivares avaliadas (Tabela 1), é possível inferir que as amostras também continham potenciais osmóticos semelhantes, e as diferenças verificadas na sensibilidade ao rachamento pelas diferentes amostras não teriam sido influenciadas pelo potencial osmótico.

No teste de compressão das bagas, verificaram-se diferenças significativas entre as cultivares (Tabela 3). A ‘BRS Rúbea’ apresentou a maior média (17,1 kPa), e os menores valores foram obtidos com a 'Concord' (6,7 kPa), tendo a cultivar Isabel apresentado valores intermediários de pressão de compressão $(10,1 \mathrm{kPa})$.

No teste de compressão, a aplicação de uma força crescente na direção axial das amostras de bagas de uva provoca um aumento da pressão interna e a deformação das bagas até o momento da ruptura da película. Lustig e Bernstein (1985) estudaram as propriedades mecânicas de bagas de uva, e na tentativa de criar um modelo consideraram as bagas como sendo pequenos reservatórios de líquido revestidos por uma fina parede onde a pressão interna seria equivalente à pressão de turgescência. Esses autores verificaram que a resistência das bagas de uva ao rompimento é uma função da tensão da casca no ponto de ruptura, da capacidade da casca em se distender e da pressão interna, principalmente da pressão de turgescência, e estes fatores variam com a maturação. Dessa forma, a pressão de compressão medida pelo texturômetro pode ser considerada equivalente à pressão interna ou à pressão de turgescência, e a compressão provocaria um efeito semelhante ao que ocorre no campo quando 
a videira e as bagas sorvem água por diferença de potencial osmótico.

Considerando que a pressão de compressão seja equivalente à pressão de turgescência, é possível afirmar que a cultivar BRS Rúbea suporta maior pressão interna antes do rompimento da película, pois apresentou a maior média de pressão de compressão no rompimento $(17,1 \mathrm{kPa})$, sendo, portanto, a cultivar mais resistente ao rachamento. A cultivar Isabel teria uma resistência média, e a 'Concord' seria, das três cultivares avaliadas, a mais suscetível ao rachamento. Estas conclusões estão de acordo com os resultados obtidos no teste de imersão, em que a 'BRS Rúbea' apresentou o menor índice de rachamento (0,0 \%), seguida pela 'Isabel' (6,3 \%) e pela 'Concord' (13,5 $\%)$. Verificou-se forte correlação negativa $(r=-0,96)$ entre o índice de rachamento e os valores de pressão de compressão obtidos (Tabela 4).

Considine (1981) determinou a pressão de turgescência para o rachamento de bagas de diversas cultivares de videira e verificou que os maiores valores (3,6 a 4,5 MPa) correspondiam aos materiais mais resistentes em condições de campo, enquanto valores menores de pressão de turgescência (1,1 a 2,0 MPa) foram obtidos com cultivares mais suscetíveis. Considine e Kriedemann (1972) também relacionaram a pressão de turgescência no momento da ruptura da película com a suscetibilidade ao rachamento e observaram que cultivares de videira mais sensíveis apresentavam valores de pressão menores (14,0 atm), ao passo que as maiores médias foram verificadas para as cultivares mais resistentes (48,9 atm). Hankinson et al. (1977) verificaram que a cultivar Thompson Seedless (Vitis vinifera) apresentou valores inferiores de pressão em relação às cultivares do grupo muscadínia (Vitis rotundifolia), que apresentam maior resistência em condições de campo.

Os valores de energia de deformação (Tabela 3) das três cultivares apresentaram diferenças significativas entre si. A 'BRS Rúbea' apresentou o maior valor, seguida pela 'Isabel' e pela 'Concord', com valores de 43,9; 25,0 e 16,7 N.mm, respectivamente.

A energia de deformação é o trabalho mecânico necessário para deformar a baga até a ruptura e fornece-nos uma medida da resistência da baga considerando a tensão máxima que a baga pode suportar antes de rachar, e sua capacidade de distender e deformar para suportar o aumento da pressão (ROSENTHAL, 1999). Sendo assim, é possível inferir que a 'BRS Rúbea', ao apresentar maior valor de energia de deformação, é capaz de suportar melhor a tensão causada pela deformação e o aumento da pressão interna, sendo, portanto, mais resistente ao rachamento. Por outro lado, a 'Concord' mostrou-se menos resistente e seus valores de energia de deformação representam sua baixa capacidade de suportar a distensão e a deformação. Os valores de energia de deformação obtidos apresentaram alta correlação negativa com o índice de rachamento $(r=-0,95)$ (Tabela 4).

Hankinson et al. (1977) compararam videiras muscadínias com a 'Thompson Seedless' e verificaram os maiores valores de energia de deformação para as cultivares mais resistentes. Sato et al. (1997), com base principalmente na energia de deformação, concluíram que as cultivares de V. labrusca são mais resistentes que as de $V$. vinifera.

Os maiores valores de força de perfuração foram observados nas cultivares BRS Rúbea e Isabel (1,29 e 1,16 N, respectivamente). A cultivar Concord apresentou a menor média de força $(0,47 \mathrm{~N})$, o que demonstra a menor resistência de sua película (Tabela 5).

Os testes de perfuração com sondas de menor calibre medem principalmente a resistência da casca dos frutos. Ao se utilizar uma sonda de conformação pontiaguda, são necessários valores menores de força para o rompimento da película, e esse tipo de sonda provoca baixa deformação. Essas observações podem ser verificadas nos resultados obtidos no teste de perfuração, principalmente em comparação com as médias de força e deformação do teste de compressão (Tabela 3). O teste de perfuração induz o rompimento da baga pela ação da ponta da sonda no ponto de contato com a película e não pelo aumento da pressão interna, como no teste de compressão. Dessa forma, é possível avaliar apenas a resistência da película de cada cultivar e relacioná-la com a resistência ao rachamento. Os resultados obtidos indicam uma importante contribuição da película para a resistência ao rachamento. Os valores de força máxima de perfuração apresentaram forte correlação negativa com o índice de rachamento $(r=-0,96)$ (Tabela 4).

Segade et al. (2008) avaliaram a textura de 13 cultivares de $V$. vinifera e verificaram valores de força máxima de perfuração variando de 0,30 a 0,66 N e não observaram correlação entre a força de perfuração e a espessura da película. Segundo Hankinson et al. (1977), a resistência à compressão está ligada à coesividade entre as células da epiderme, enquanto a resistência à perfuração está relacionada ao tamanho destas células. Letaief et al. (2006) obtiveram valores de força de perfuração para as cultivares Cabernet Sauvignon (0,46 N), Pinot Noir (0,50 N) e Nebbiolo $(0,35 \mathrm{~N})$. Fava et al. (2011) encontraram valores de 4,9 N para força máxima de perfuração da cultivar Isabel, superior ao obtido no presente trabalho, 
porém utilizaram uma sonda de três milímetros de diâmetro com ponta de seção plana e não do tipo agulha, o que pode ter provocado um efeito parcial de compressão. Dessa forma, a ruptura da película não teria sido provocada apenas pela perfuração da casca, mas também pelo aumento da pressão interna, como no teste de compressão. Wu e Abbott (2002) verificaram diferenças significativas nos valores de força quando usaram sondas de um e de três milímetros em testes de perfuração em tomate.

Para o teste de relaxação, os valores do grau de solidez (1- $\left.\mathrm{C}_{1}\right)$ e da velocidade de decaimento $\left(\mathrm{C}_{1} /\right.$ $\mathrm{C}_{2}$ ) são apresentados na Tabela 5. Quanto ao grau de solidez, a cultivar Concord diferiu das demais, apresentando a maior média $(0,722)$, enquanto a Isabel e BRS Rúbea apresentaram médias inferiores e semelhantes entre si $(0,671$ e 0,674 , respectivamente). Os maiores valores de velocidade inicial de decaimento foram obtidos para as cultivares Isabel e BRS Rúbea (0,297 e 0,308, respectivamente), sendo a média verificada para 'Concord' inferior às demais.

Os testes de relaxação estática em bagas de uva mostram-nos as características dos componentes elásticos e viscosos de cada material. As características viscoelásticas dos frutos da videira foram observadas nas cultivares avaliadas e foram descritas por outros autores (HANKINSON et al., 1977; LUSTIG; BERNSTEIN, 1985).

Quando o grau de solidez tende a um, o material comporta-se mais como um sólido elástico, e quando tende a zero é controlado pelo componente viscoso do material. Verifica-se que a 'Concord' é mais elástica que as demais quando submetida a uma deformação constante, e sua estrutura tende a ceder menos à deformação imposta. Por outro lado, a
'Isabel' e 'BRS Rúbea’ apresentaram comportamento um pouco mais viscoso, ou seja, sua estrutura e seus tecidos tendem a acomodar-se mais à deformação imposta pela aplicação da força. Segundo Moghimi et al. (2011), as diferenças entre o comportamento viscoelástico dos frutos ocorrem por diferenças de firmeza e coesão dos tecidos estruturais.

A velocidade inicial de decaimento é inversamente proporcional ao grau de solidez. Maiores valores de velocidade de decaimento correspondem a maior escoamento ou maior viscosidade, e valores menores indicam maior elasticidade.

A média de velocidade de decaimento verificada para 'Concord' foi inferior, confirmando o comportamento mais elástico já verificado pelo grau de solidez dessa cultivar. A 'Isabel' e a 'BRS Rúbea', ao apresentarem valores de velocidade inicial de decaimento superiores, demonstram maior capacidade de acomodação das células e dos tecidos de suas estruturas ao aumento da tensão provocada pela compressão estática. Dessa forma, pode-se inferir que estes materiais, ao aumentarem seu volume de baga e pressão de turgescência pela sorção de água no campo, teriam maior resistência ao rachamento, pois a viscosidade ajudaria a suportar essa variação no volume sem que ocorresse o rompimento da película da baga. Para os valores de grau de solidez, foi verificada forte correlação com o índice de rachamento $(r=0,88)$, assim como para a velocidade de decaimento $(r=-0,95)$ (Tabela 4$)$.

TABELA 1 - Teor de sólidos solúveis - SS ( ${ }^{\circ}$ Brix) e acidez titulável - AT (\% de ácido tartárico) do mosto de bagas de videiras das cultivares Concord, Isabel e BRS Rúbea, no momento da colheita. Rolândia-PR, 2011.

\begin{tabular}{lcc}
\hline Cultivar & SST $\left({ }^{\circ}\right.$ Brix) & AT (\%) \\
\hline Concord & $12,68 \mathrm{a} \pm 0,13$ & $0,84 \mathrm{a} \pm 0,05$ \\
Isabel & $13,00 \mathrm{a} \pm 0,18$ & $0,70 \mathrm{a} \pm 0,03$ \\
BRS Rúbea & $13,08 \mathrm{a} \pm 0,12$ & $0,47 \mathrm{~b} \pm 0,03$ \\
\hline CV $(\%)$ & 2,52 & 12,69 \\
\hline
\end{tabular}

Médias seguidas pelas mesmas letras nas colunas diferem entre si, pelo teste de Tukey $(P<0,05)$. 
TABELA 2 - Índice de rachamento de bagas de videiras das cultivares Concord, Isabel e BRS Rúbea. Rolândia-PR, 2011.

\begin{tabular}{lccc}
\hline Cultivar & Total de bagas rachadas & Valor ponderado & Índice de rachamento (\%) \\
Concord & 8,5 & 67,5 & $13,5 \mathrm{a} \pm 0,1$ \\
Isabel & 5,0 & 31,5 & $6,3 \mathrm{~b} \pm 1,1$ \\
BRS Rúbea & 0,0 & 0,0 & $0,0 \mathrm{c} \pm 0,0$ \\
\hline $\mathrm{CV}(\%)$ & & & 13,66 \\
\hline
\end{tabular}

Médias seguidas pelas mesmas letras nas colunas diferem entre si, pelo teste de Tukey $(P<0,05)$.

TABELA 3 - Pressão de compressão, energia de deformação e força máxima de compressão de bagas de videiras das cultivares Concord, Isabel e BRS Rúbea. Rolândia-PR, 2011.

\begin{tabular}{lccc}
\hline Cultivar & Pressão de & $\begin{array}{c}\text { Energia de deformação } \\
(\mathrm{N} . \mathrm{mm})\end{array}$ & $\begin{array}{c}\text { Força máxima de } \\
\text { compressão }(\mathrm{N})\end{array}$ \\
Concord & $6,7 \mathrm{c} \pm 0,4$ & $16,7 \mathrm{c} \pm 0,6$ & $6,8 \mathrm{c} \pm 0,4$ \\
Isabel & $10,1 \mathrm{~b} \pm 0,6$ & $25,0 \mathrm{~b} \pm 1,3$ & $10,3 \mathrm{~b} \pm 0,6$ \\
BRS Rúbea & $17,1 \mathrm{a} \pm 0,9$ & $43,9 \mathrm{a} \pm 1,6$ & $17,4 \mathrm{a} \pm 0,9$ \\
\hline CV $(\%)$ & 22,24 & 15,12 & 22,23 \\
\hline
\end{tabular}

Médias seguidas pelas mesmas letras nas colunas diferem entre si, pelo teste de Tukey $(P<0,05)$.

TABELA 4 - Correlações entre o índice de rachamento e as variáveis dos testes mecânicos para bagas de videiras das cultivares Concord, Isabel e BRS Rúbea. Rolândia-PR, 2011.

\section{Relações}

Índice de rachamento - Pressão de compressão

Índice de rachamento - Energia de deformação

Índice de rachamento - Força de Perfuração

Índice de rachamento - Grau de solidez

Índice de rachamento - Velocidade de decaimento
Coeficiente de correlação de Pearson (r)

$$
\begin{array}{r}
-0,96 \\
-0,95 \\
-0,96 \\
0,88 \\
-0,95
\end{array}
$$

TABELA 5 - Força máxima de perfuração, deformação de perfuração grau de solidez e velocidade de decaimento de bagas de videiras das cultivares Concord, Isabel e BRS Rúbea. Rolândia-PR, 2011.

\begin{tabular}{lcccc}
\hline Cultivar & $\begin{array}{c}\text { Força máxima } \\
\text { de perfuração }(\mathrm{N})\end{array}$ & $\begin{array}{c}\text { Deformação } \\
\text { de perfuração }(\%)\end{array}$ & $\begin{array}{c}\text { Grau de solidez } \\
\left(1-\mathrm{C}_{1}\right)\end{array}$ & $\begin{array}{c}\text { Velocidade de decaimento } \\
\left(\mathrm{C}_{1} / \mathrm{C}_{2}\right)\end{array}$ \\
Concord & $0,47 \mathrm{~b} \pm 0,05$ & $2,66 \mathrm{~b} \pm 0,26$ & $0,722 \mathrm{a} \pm 0,002$ & $0,220 \mathrm{~b} \pm 0,006$ \\
Isabel & $1,16 \mathrm{a} \pm 0,06$ & $6,45 \mathrm{a} \pm 0,34$ & $0,671 \mathrm{~b} \pm 0,003$ & $0,297 \mathrm{a} \pm 0,009$ \\
BRS Rúbea & $1,29 \mathrm{a} \pm 0,04$ & $7,18 \mathrm{a} \pm 0,21$ & $0,674 \mathrm{~b} \pm 0,003$ & $0,308 \mathrm{a} \pm 0,008$ \\
\hline CV (\%) & 19,51 & 19,52 & 1,55 & 10,88 \\
\hline
\end{tabular}

Médias seguidas pelas mesmas letras nas colunas diferem entre si,pelo teste de Tukey $(P<0,05)$.

\section{CONCLUSÕES}

1-As cultivares BRS Rúbea, Concord e Isabel apresentam diferenças com relação à suscetibilidade ao rachamento de bagas na maturação, tanto pelo teste de imersão como pelos testes mecânicos. A cultivar BRS Rúbea é a mais resistente ao rachamento, e a cultivar Concord, a mais suscetível. A 'Isabel' apresenta resistência intermediária.
2-O índice de rachamento obtido pelo teste de imersão apresenta boa correlação com as variáveis avaliadas nos testes mecânicos e, por sua simplicidade e facilidade de execução, possui bom potencial para avaliação da sensibilidade ao rachamento de cultivares de videira. 


\section{AGRADECIMENTOS}

À Corol Cooperativa Agroindustrial, à Universidade Estadual de Londrina, à Empresa Brasileira de Pesquisa Agropecuária, ao Conselho Nacional de Desenvolvimento Científico e Tecnológico e aos colegas dos Departamentos de Agronomia e Ciência e Tecnologia de Alimentos da UEL.

\section{REFERÊNCIAS}

BERNSTEIN, Z.; LUSTIG, I. A new method of firmness measurement on grape berries and other juicy fruits. Vitis, Siebeldingen, v.20, p.15-21, 1981.

BERNSTEIN, Z.; LUSTIG, I. Hydrostatic methods of measurement of firmness and turgor pressure of grape berries (Vitis vinifera L.). Scientia Horticulturae, Amsterdam, v.25, p.129-136, 1985.

CALBO, A. G.; NERY, A. A. Medida de firmeza de hortaliças pela técnica de aplanação. Horticultura Brasileira, Brasília, v.13, n.1, p.14-18, 1995.

CAMARGO, U. A.; MAIA, J. D. G.; RITSCHEL, P. S. Novas cultivares brasileiras de uva. Bento Gonçalves: Embrapa Uva e Vinho, 2010. 64 p.

CLARKE, S. J.; HARDIE, W. J.; ROGIERS, S. Y. Changes in susceptibility of grape berries to splitting are related to impaired osmotic water uptake associated with losses in cell vitality. Australian Journal of Grape and Wine Research, Adelaide, v.16, n.3, p.469-476, 2010.

CONSIDINE, J. A. Correlation of resistance to physical stress with fruit structure in the grape Vitis vinifera L. Australian Journal of Botany, Melbourne, v.29, n.2, p.475 - 482, 1981.

CONSIDINE, J. A. Physical aspects of fruit growth: cuticular fracture and fracture patterns in relation to fruit structure in Vitis vinifera. Journal of Horticultural Science, Ashford, v.57, n. 1, p.7991, 1982.

CONSIDINE, J. A.; BROWN, K. Physical aspects of fruit growth: Theoretical analisis of distribution of surface growth forces in fruit in relation to cracking and splitting. Plant Physiology, Rockville, v.68, p.371-376, 1981.
CONSIDINE, J. A.; KRIEDMAN, P. E. Fruit splitting in grapes: Determination of the critical turgor pressure. Australian Journal of Agricultural Research, Queensland, v.23, n.1, p.17-24, 1972.

FAVA, J.; HODARA, K.; NIETO, A.; GUERRERO, S.; ALZAMORA, S. M.; CASTRO, M. A. Structure (micro, ultra, nano), color and mechanical properties of Vitis labrusca L. (grape berry) fruits treated by hydrogen peroxide, UV-C irradiation and ultrasound. Food Research International, Guelph, v.44, n.9, p.2.938-2.948, 2011.

HANKINSON, B.; RAO, V. N. M.; SMIT, C. J. B. Viscoelastic and histological properties of grape skins. Journal of Food Science, Chicago, v.42, n.3, p.632-635, 1977.

LANG, A.; DÜRING, H. Grape berry splitting and some mechanical properties of the skin. Vitis, Siebeldingen, v.29, n.1, p.61-70, 1990.

LETAIEF, H.; ROLLE, L.; ZEPPA, G.; GERBI, V. Grape skin and seeds hardness assessment by texture analysis. In: WORLD CONGRESS OF FOOD SCIENCE \& TECHNOLOGY, 13., 2006; Nantes. Anais... p.1877-1896.

LULU, J.; CASTRO, J.; PEDRO JÚNIOR, M. J. Efeito do microclima na qualidade da uva de mesa 'Romana' (A 1105) cultivada sob cobertura plástica. Revista Brasileira de Fruticultura, Jaboticabal, v.27, n.3, p.422-425, 2005.

LUSTIG, I.; BERNSTEIN, Z. Determination of the mechanical properties of the grape berry skin by hydraulic measurements. Scientia Horticulturae, Amsterdam, v.25, n.1, p.279-285, 1985.

MATTHEWS, M. A.; CHENG, G.; WEINBAUM, S. A. Changes in water potential and dermal extensibility during grape berry development. Journal of the American Society for Horticultural Science, Alexandria, v.112, n.2, p.314-319, 1987.

MELlO, L. M. R. de. Atuação do Brasil no mercado vitivinícola mundial: panorama 2009. Bento Gonçalves: EMBRAPA/CNPUV, 2009. Disponível em: < http://www.embrapa.cnpuv.br>. Acesso em: 05 dez. 2011. 
MOGHIMI, A.; SAIEDIRAD, M. H.; MOGHADAM, E. G. Interpretation of viscoelastic behaviour of sweet cherries using rheological models. International Journal of Food Science \& Technology, Manchester; v.46, p.855-861, 2011.

NACHTIGAL, J. C.; SCHNEIDER, E. P. Recomendações para produção de videiras em sistemas de base ecológica. Bento Gonçalves: Embrapa Uva e Vinho, 2007. 67 p. (Documentos, 65).

PELEG, M. Characterization of stress relaxation curves of solid food. Journal of Food Science, Chicago, v.44, p.277-280, 1979.

ROSENTHAL, A. J. Food texture: measurement and perception. London: Chapman \& Hall, 1999. 311p.

SATO, A. J.; SILVA, B. J. da; SANTOS, C. E. dos; BERTOLUCCI, R.; SANTOS, R. dos; CARIELO, M.; GUIRAUD, M. C.; FONSECA, I. C. de B.; ROBERTO, S. R. Características físico-químicas e produtivas das uvas 'Isabel' e 'BRS-Rúbea' sobre diferentes porta-enxertos na região norte do Paraná. Revista Brasileira de Fruticultura, Jaboticabal, v.30, n.2, p.553-556, 2008.

SATO, A.; YAMANE, H.; HIRAKAWA, N.; OTOBE, K.; YAMADA, M. Varietal differences in the texture of grape berries measured by penetration tests. Vitis, Siebeldingen, v.36, n.1, p.7-10, 1997.
SEGADE, S. R.; ROLLE, L.; GERBI, V.; ORRIOLS, I. Phenolic ripeness assessment of grape skin by texture analysis. Journal of Food Composition and Analysis, Rome, v.21, n.8, p. 644-649, 2008.

STATSOFT. Statistica for windows: . Computer program manual. Tulsa: StatSoft, 2002

THOMAS, T. R.; SHACKEL, K. A.; MATTHEWS, M. A. Mesocarp cell turgor in Vitis vinifera L. berries throughout development and its relation to firmness, growth, and the onset of ripening. Planta, New York, v.228, p.1.067-1.076, 2008.

VERNER, L.; BLODGETT, E. C. Physiological studies of the cracking of sweet cherries., Moscow: University of Idaho Agricultural Experiment Station, 1936. 15 p. (Bulletin,184)

WU, T.; ABBOTT, J. A. Firmness and force relaxation characteristics of tomatoes stored intact or as slices. Postharvest Biology and Technology, Wageningen, v.24, n.1, p.59-68, 2002.

YAMAMOTO, T.; SATOH, H. Relationship among berry cracking susceptibility, berry morphology and skin stress distribution in several grape cultivars. Journal of the Japanese Society for Horticultural Science, Kyoto, v.63, n.2, p.247-256, 1994. 